\title{
Decreased expression of haem oxygenase-1 by alveolar macrophages in idiopathic pulmonary fibrosis
}

\author{
Q. Ye*,\#, Y. Dalavanga*, N. Poulakis*, S.U. Sixt ", J. Guzman ${ }^{+}$and U. Costabel*
}

ABSTRACT: Haem oxygenase (HO)-1 is an oxidative stress responsive protein that may be involved in the pathogenesis of interstitial lung disease.

HO-1 expression in alveolar macrophages from bronchoalveolar lavage was investigated in 24 patients with idiopathic pulmonary fibrosis (IPF), 16 with sarcoidosis, 14 with hypersensitivity pneumonitis (HP) and 13 controls. Using immunocytochemistry, HO-1 expression in macrophages was scored semiquantitatively from 0-3 according to increasing intensity. The mean score of 100 macrophages was calculated. Macrophages were cultured and levels of interleukin (IL)-12 and IL-18 in the culture supernatants were measured by ELISA.

The mean score of HO-1 was significantly lower in IPF (67) than in sarcoidosis (105), HP (106) or controls (106). There was no significant difference between sarcoidosis, HP and controls. The score of HO-1 correlated positively with the lymphocyte percentage in sarcoidosis and HP. Positive correlations were found between the score of HO-1 and the release of IL-12 and IL-18 by macrophages in IPF.

The expression of haem oxygenase-1, a critical defender against oxidative stress, is decreased in macrophages of idiopathic pulmonary fibrosis patients compared with those with granulomatous lung disorders. This supports the hypothesis of an oxidant-antioxidant imbalance in the pathogenesis of idiopathic pulmonary fibrosis.

\section{KEYWORDS: Alveolar macrophage, cytokine, haem oxygenase-1, interstitial lung disease}

diopathic pulmonary fibrosis (IPF) is a chronic and fatal lung disease of unknown cause characterised by progressive fibroblast proliferation, destruction of the alveolar architecture and a relentless decline in pulmonary function [1]. The precise pathogenetic mechanisms of IPF are yet to be determined. A number of studies have suggested that an oxidant-antioxidant imbalance plays a role in the progression of pulmonary fibrosis in animal models and also possibly in human IPF [2]. There is ample evidence suggesting increased oxidative stress in patients with IPF. Alveolar macrophages (AMs) and neutrophils, which dominate in the lower respiratory tract of patients with IPF, are capable of inducing oxidant-mediated injury to lung parenchymal cells $[3,4]$. Superoxide radicals and hydrogen peroxide are released spontaneously by bronchoalveolar lavage (BAL) cells of IPF patients [3]. High levels of myeloperoxidase associated with epithelial injury were found in the alveolar epithelial lining fluid (ELF) and BAL fluid (BALF) of patients with IPF [3]. Oxidative modification of mitochondrial
DNA has been observed in lung epithelial cells of IPF patients [5]. Oxidised BALF proteins, characterised by oxidation of methionine residues or carbonylation, are increased in patients with IPF $[6,7]$. There is also an increase in 8 -isoprostane, a biomarker of oxidative stress, in the BALF of IPF patients [8].

Conversely, there is a deficiency of cellular antioxidants in IPF patients [9-12]. Glutathione, a major component of the lung antioxidant defence system, is decreased in the ELF, both in the bleomycin model of lung fibrosis and in IPF patients [9]. Antioxidants and detoxification enzymes, including thioredoxin, glutaredoxin, manganese superoxide dismutase (MnSOD), catalase and glutamate cysteine ligase ( $\gamma$-glutamyl cysteine synthetase, the rate-limiting enzyme in glutathione synthesis), show a diminished expression in patients with IPF [10-12]. These data support the hypothesis of an oxidantantioxidant imbalance in the pathogenesis of IPF.

Haem oxygenase $(\mathrm{HO})$, the rate-limiting enzyme in the catabolism of haem, is responsible for the

\section{AFFILIATIONS}

*Dept of Pneumology and

Allergology, Ruhrlandklinik, Medical Faculty,

-Dept of Anesthesiology and Intensive Care Medicine, School of Medicine Essen, University of Duisburg-Essen, Essen, ${ }^{+}$General and Experimental Pathology, Ruhr University, Bochum, Germany.

${ }^{\#}$ Beijing Institute of Respiratory Medicine, Beijing Chaoyang Hospital, Capital University of Medical Sciences, Beijing, China.

\section{CORRESPONDENCE}

U. Costabel

Dept of Pneumology and Allergology Ruhrlandklinik

Tüschener Weg 40

45239 Essen

Germany

Fax: 492014332009

E-mail: ulrich.costabel@

ruhrlandklinik.de

Received:

September 242007

Accepted after revision:

January 042008

SUPPORT STATEMENT

This work was supported by the Arbeitsgemeinschaft zur Förderung der Pneumologie an der Ruhrlandklinik (AFPR; Essen, Germany).

STATEMENT OF INTEREST None declared. 
TABLE 1 Demographics of the study population

\begin{tabular}{|c|c|c|c|c|}
\hline & IPF & Sarcoidosis ${ }^{\#}$ & HP" & Controls \\
\hline Subjects (M/F) n & $24(20 / 4)$ & $16(7 / 9)$ & $14(8 / 6)$ & $13(8 / 5)$ \\
\hline Age yrs & $67 \pm 2^{* \star *}$ & $48 \pm 4^{*,+, \S}$ & $61 \pm 4^{\star \star *}$ & $37 \pm 3$ \\
\hline Nonsmokers $\mathrm{n}$ & 14 & 16 & 10 & 13 \\
\hline Current smokers n & 0 & 0 & 0 & 0 \\
\hline Ex-smokers n & 10 & 0 & 4 & 0 \\
\hline VC \% pred & $72 \pm 4^{\star \star}$ & $80 \pm 6$ & $70 \pm 7^{\star \star}$ & $99 \pm 4$ \\
\hline TLC \% pred & $68 \pm 3^{* \star *}$ & $79 \pm 4$ & $78 \pm 5$ & $97 \pm 4$ \\
\hline $\mathrm{FEV}_{1} / \mathrm{VC} \%$ & $77 \pm 2$ & $74 \pm 2$ & $75 \pm 2$ & $85 \pm 5$ \\
\hline $\mathrm{Pa}, \mathrm{O}_{2} \mathrm{mmHg}$ & $71 \pm 2^{\star \star \star}$ & $77 \pm 2$ & $73 \pm 4^{* *}$ & $90 \pm 2$ \\
\hline $\mathrm{PA}-\mathrm{a}, \mathrm{O}_{2} \mathrm{mmHg}$ & $30 \pm 3^{\star \star \star}$ & $21 \pm 2$ & $32 \pm 5^{\star \star}$ & $11 \pm 1$ \\
\hline
\end{tabular}

Data expressed as mean \pm SEM, unless otherwise stated. IPF: idiopathic pulmonary fibrosis; HP: hypersensitivity pneumonitis; M: male; F: female; VC: vital capacity; \% pred: \% predicted; TLC: total lung capacity; FEV1: forced expiratory volume in one second; $\mathrm{Pa}_{1} \mathrm{O}_{2}$ : arterial oxygen tension; $\mathrm{PA}-\mathrm{a}, \mathrm{O}_{2}$ : alveolar-arterial oxygen tension difference. ${ }^{\#}$ : according to chest radiographical staging, six sarcoidosis patients had stage I, six patients had stage II and four patients had stage III disease; ": six were budgerigar fanciers, seven were pigeon breeders and one had humidifier lung. ${ }^{*}: p<0.05,{ }^{* *}$ : $p<0.01$ and ${ }^{* *}$. $p<0.001$ (when compared with controls); ${ }^{+}: p<0.001$ when compared with IPF s: $\mathrm{p}<0.01$ when compared with HP. $1 \mathrm{mmHg}=0.133 \mathrm{kPa}$

degradation of haem to biliverdin, free iron, and carbon monoxide (CO). Biliverdin is subsequently converted to bilirubin through the action of the cytosolic enzyme biliverdin reductase, and free iron is sequestered by ferritin [13]. At present, three isoforms of $\mathrm{HO}$ have been identified as HO-1, $\mathrm{HO}-2$ and $\mathrm{HO}-3$. HO-1, the inducible form of $\mathrm{HO}$, is thought to be an oxidative stress responsive protein, whereas $\mathrm{HO}-2$ and HO-3 are constitutively expressed [13]. HO-1 has been implicated in a number of conditions and diseases associated with lung injury. HO-1 seems to be upregulated in smokers, asthma, acute lung injury, cystic fibrosis and lung transplant rejection, whereas it is downregulated in severe chronic obstructive pulmonary disease [14-19]. Since an oxidantantioxidant imbalance contributes to the pathogenesis of lung fibrosis, HO-1, one of the critical defenders against oxidative stress, may be important in fibrotic lung diseases.

The aim of the present study was to investigate the expression of HO-1 in AMs recovered by BAL in patients with IPF, sarcoidosis and hypersensitivity pneumonitis (HP). Potential correlations between the expression of $\mathrm{HO}-1$ and the production of pro-inflammatory cytokines were also explored. For this purpose, interleukin (IL)-12 and IL-18 were chosen, since they are involved in regulating the balance between T-helper cell (Th) type 1 (Th1) and Th type 2 (Th2) profiles [20, 21]. IPF is thought to be associated with a Th2 profile [22], whereas sarcoidosis and HP are Th1 diseases [23, 24].

\section{METHODS}

\section{Patients}

In the present study, 24 consecutive patients with IPF, 16 patients with active pulmonary sarcoidosis, 14 with chronic $\mathrm{HP}$ and 13 control subjects were investigated (table 1).
No patient was receiving treatment with corticosteroids and/ or immunosuppressants at the time of BAL. Written informed consent was obtained according to institutional guidelines.

The 24 IPF patients were diagnosed according to American Thoracic Society/European Respiratory Society criteria [1]. Of these, seven had a surgical biopsy with histological evidence of usual interstitial pneumonia (UIP).

The 16 sarcoidosis patients were diagnosed on the basis of compatible clinical and radiographical features, histological evidence of noncaseating granuloma and/or an increased CD4/CD8 ratio of BAL lymphocytes, and the exclusion of other granulomatous disorders [23]. All of the sarcoidosis patients showed manifestations of disease activity as evidenced by recently developed or worsening symptoms, and/ or chest radiographical evidence of progressive disease and/or deterioration of lung function tests.

The $14 \mathrm{HP}$ patients fulfilled the following diagnostic criteria: 1) a history of exposure to organic antigens; 2) clinical signs and symptoms consistent with $\mathrm{HP}$; 3) radiographical features and/or functional abnormalities characteristic of interstitial lung disease; 4) evidence of serum precipitins against one or more organic antigens; and 5) increased lymphocytes in the BALF. All $14 \mathrm{HP}$ patients presented with the chronic form of insidious onset. On high-resolution computed tomography, all HP patients showed widespread and dominant ground-glass densities, with only minor reticulation and no honeycombing. Late-stage cases with extensive fibrosis were not investigated in the present study.

\begin{tabular}{|c|c|c|c|c|}
\hline & IPF & Sarcoidosis & HP & Controls \\
\hline \multicolumn{5}{|l|}{ Total cells } \\
\hline $\mathrm{n} \times 10^{6}$ & $15.0 \pm 2.4$ & $17.0 \pm 2.6^{*}$ & $25.3 \pm 3.7^{\star \star \star}, \#$ & $6.9 \pm 1.0$ \\
\hline $\mathrm{n} \times 10^{4} \cdot \mathrm{mL}^{-1}$ & $8.1 \pm 1.1$ & $12.0 \pm 1.4^{*}$ & $19.3 \pm 3.5^{\star \star \star \star \# \# \# ~}$ & $5.8 \pm 0.9$ \\
\hline \multicolumn{5}{|l|}{ Macrophages } \\
\hline$\%$ & $76.3 \pm 3.3$ & $52.9 \pm 5.4^{\star \star \star}$ & 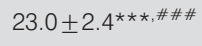 & $92.1 \pm 0.9$ \\
\hline $\mathrm{n} \times 10^{4} \cdot \mathrm{mL}^{-1}$ & $6.2 \pm 1.0$ & $5.8 \pm 0.7$ & $4.5 \pm 1.0$ & $5.3 \pm 0.8$ \\
\hline \multicolumn{5}{|l|}{ Lymphocytes } \\
\hline$\%$ & $14.0 \pm 2.6$ & 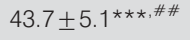 & 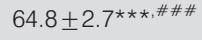 & $6.8 \pm 0.9$ \\
\hline $\mathrm{n} \times 10^{4} \cdot \mathrm{mL}^{-1}$ & $1.3 \pm 0.4$ & $5.8 \pm 1.0^{\star *, \# \#}$ & 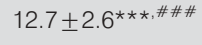 & $0.4 \pm 0.09$ \\
\hline \multicolumn{5}{|l|}{ Granulocytes } \\
\hline$\%$ & $9.7 \pm 1.5^{\star \star \star}$ & $3.7 \pm 0.8^{\bullet}$ & $11.8 \pm 2.2^{\star \star \star}$ & $1.2 \pm 0.3$ \\
\hline $\mathrm{n} \times 10^{4} \cdot \mathrm{mL}^{-1}$ & $0.7 \pm 0.1$ & $0.5 \pm 0.1^{\star \star \star \star * \# \#}$ & $2.1 \pm 0.5^{\star \star \star *, \#}$ & $0.07 \pm 0.02$ \\
\hline \multicolumn{5}{|l|}{ Neutrophils } \\
\hline$\%$ & $7.0 \pm 1.2^{\star * *}$ & $2.5 \pm 0.6$ & $8.5 \pm 2.0^{\star \star \star}$ & $1.1 \pm 0.3$ \\
\hline $\mathrm{n} \times 10^{4} \cdot \mathrm{mL}^{-1}$ & $0.5 \pm 0.09$ ** & $0.3 \pm 0.08 * *$. & $1.6 \pm 0.5^{\star \star \star}$ & $0.07 \pm 0.02$ \\
\hline \multicolumn{5}{|l|}{ Eosinophils } \\
\hline$\%$ & $2.5 \pm 0.5^{\star \star \star}$ & $0.9 \pm 0.3$ & $2.6 \pm 0.7^{\star \star \star}$ & $0.08 \pm 0.03$ \\
\hline $\mathrm{n} \times 10^{4} \cdot \mathrm{mL}^{-1}$ & $0.2 \pm 0.03^{* \star *}$ & $0.1 \pm 0.05^{\star \star}$ & $0.4 \pm 0.08^{\star * \star}$ & $0.001 \pm 0.00$ \\
\hline
\end{tabular}

Data expressed as mean \pm SEM. IPF: idiopathic pulmonary fibrosis; HP: hypersensitivity pneumonitis. ${ }^{*}: p<0.05,{ }^{* *}: p<0.01$ and ${ }^{* *}: p<0.001$ when compared with controls; ${ }^{*}: p<0.05,{ }^{\# \#:}: p<0.01$ and ${ }^{\# \# \#: ~} p<0.001$ when compared with IPF; ${ }^{\imath}: p<0.05$ when compared with HP. 


\begin{tabular}{ll} 
TABLE 3 & $\begin{array}{l}\text { Semiquantitative score of haem oxygenase-1 } \\
\text { expression in bronchoalveolar lavage } \\
\text { macrophages }\end{array}$ \\
\hline Score & Staining intensity \\
\hline $\mathbf{0}$ & No staining \\
$\mathbf{2}$ & Weak staining in the cytoplasm \\
$\mathbf{3}$ & Moderate staining in the cytoplasm, nucleus still visible \\
\hline
\end{tabular}

In total, 13 patients underwent diagnostic bronchoscopy and showed no evidence of lung disease. They had normal BAL cytology and served as controls.

\section{BAL procedure}

BAL was performed during fibreoptic bronchoscopy according to established guidelines [25]. Sterile isotonic saline solution was instilled into the right middle or left ligular lobe in $20 \mathrm{~mL}$ aliquots to a total volume of $100-200 \mathrm{~mL}$, with immediate aspiration by gentle suction after each aliquot. A volume of $>50 \%$ was retrieved. The BAL cell differentials of the four study groups are shown in table 2 .

\section{Immunocytochemical analysis}

Immunocytochemistry was used to investigate the expression of HO-1 (Stressgen Biotechnologies Corp., Victoria, BC, Canada) in AMs from BAL. The peroxidase-antiperoxidase method was applied as previously described [26]. To evaluate the percentage of $\mathrm{HO}-1$ positive cells, 500 macrophages were counted under a light microscope. In addition, HO-1 expression by AMs was scored semiquantitatively. The score for each AM counted ranged $0-3$ according to increasing intensity (table 3). The mean score of 100 AMs was calculated for each patient and ranged 0-300. All analyses, including immunocytochemical grading, were performed blind by two investigators. The interobserver correlation was good. The Spearman's $r$-value for the correlation between the two counts was 0.89 $(\mathrm{p}<0.01)$.

\section{Culture of AMs}

AM cultures were performed as previously described [21], in patients with IPF $(n=12)$, sarcoidosis $(n=8)$ and HP $(n=4)$. The AMs from BAL were incubated for $24 \mathrm{~h}$ with $1 \mathrm{~mL}$ RPMI 1640 medium (Seromed; Biochrom KG, Berlin, Germany) alone, or RPMI 1640 medium with $100 \mathrm{ng} \cdot \mathrm{mL}^{-1}$ lipopolysaccharide (LPS; Sigma-Aldrich, Munich, Germany). The culture supernatants were centrifuged and stored at $-80^{\circ} \mathrm{C}$.

\section{ELISA assay for cytokines}

The concentration of IL-12p70 and IL-18 in the culture supernatants was quantified using commercially available human ELISA kits (R\&D systems, Inc., Minneapolis, MN, USA and Medical and Biological Laboratories Ltd, Nagoya, Japan), with a sensitivity of $0.5 \mathrm{pg} \cdot \mathrm{mL}^{-1}$ and $12.5 \mathrm{pg} \cdot \mathrm{mL}^{-1}$, respectively. The concentrations of these cytokines were expressed as $\mathrm{pg} \cdot \mathrm{mL}^{-1}$ per $10^{6} \mathrm{AM}$ after correction for the proportion of AMs.

\section{Total bilirubin and ferritin analysis}

The supernatants were analysed using ELISA kits for total bilirubin (Beckman Coulter, Fullerton, CA, USA) and for ferritin (Immulite; Siemens Healthcare Diagnostics, Tarrytown, $\mathrm{NY}$, USA), with sensitivities of $0.1 \mathrm{mg} \cdot \mathrm{mL}^{-1}$ and $0.4 \mathrm{ng} \cdot \mathrm{mL}^{-1}$, respectively, using the manufacturers' instructions.

\section{Statistical analysis}

Data are expressed as mean \pm SEM. To compare the four study populations (IPF, sarcoidosis, HP and control subjects), the data were analysed using Kruskal-Wallis one-way ANOVA by ranks. Two groups were compared using the unpaired t-test for parametric data. The correlations of different parameters were analysed by Spearman rank. A p-value $<0.05$ was considered statistically significant.

\section{RESULTS}

\section{Expression of $\mathrm{HO}-1$}

HO-1 immunostaining was observed mainly in BAL AMs, occasionally in epithelial cells, but not in other BAL cells including lymphocytes, neutrophils, eosinophils, mast cells and plasma cells.

The score (mean \pm SEM) of HO-1 positive AMs was significantly lower in patients with IPF $(67.4 \pm 5.0)$ than in those with sarcoidosis (105.4 $\pm 7.1, \mathrm{p}<0.01), \mathrm{HP}(105.6 \pm 5.4, \mathrm{p}<0.01)$ or control subjects $(105.9 \pm 5.2, \mathrm{p}<0.01$; fig. 1$)$. The percentage of $\mathrm{HO}-1$ positive macrophages was also significantly decreased in patients with IPF $(56 \pm 3)$ compared with those with sarcoidosis $(76 \pm 3, \mathrm{p}<0.01), \mathrm{HP}(74 \pm 3, \mathrm{p}<0.01)$ and control subjects $(86 \pm 2, \mathrm{p}<0.01)$. There was no difference in the score or percentage of $\mathrm{HO}-1$ positive macrophages between sarcoidosis and HP patients or controls.

\section{IL-12 and IL-18 release from BAL macrophages in IPF and granulomatous lung disorders}

Since the number of BAL samples with sufficient cells for AM culture was small, the patients with sarcoidosis $(n=8)$ and HP $(n=4)$ were grouped together as granulomatous lung disorders

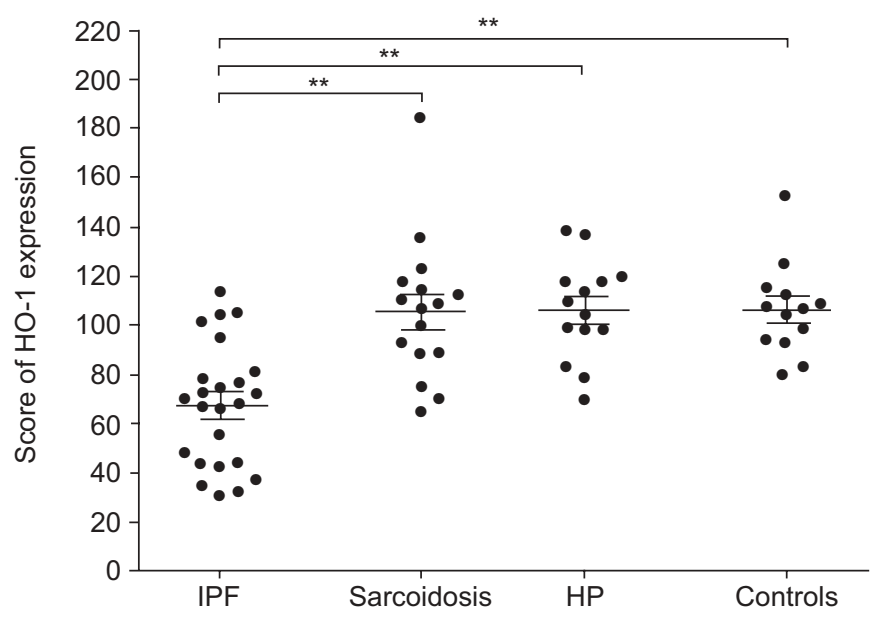

FIGURE 1. Semiquantitative score of haem oxygenase (HO)-1 expression in alveolar macrophages in bronchoalveolar lavage fluid, in patients with idiopathic pulmonary fibrosis (IPF), sarcoidosis, hypersensitivity pneumonitis (HP) and controls. **: $p<0.01$. 

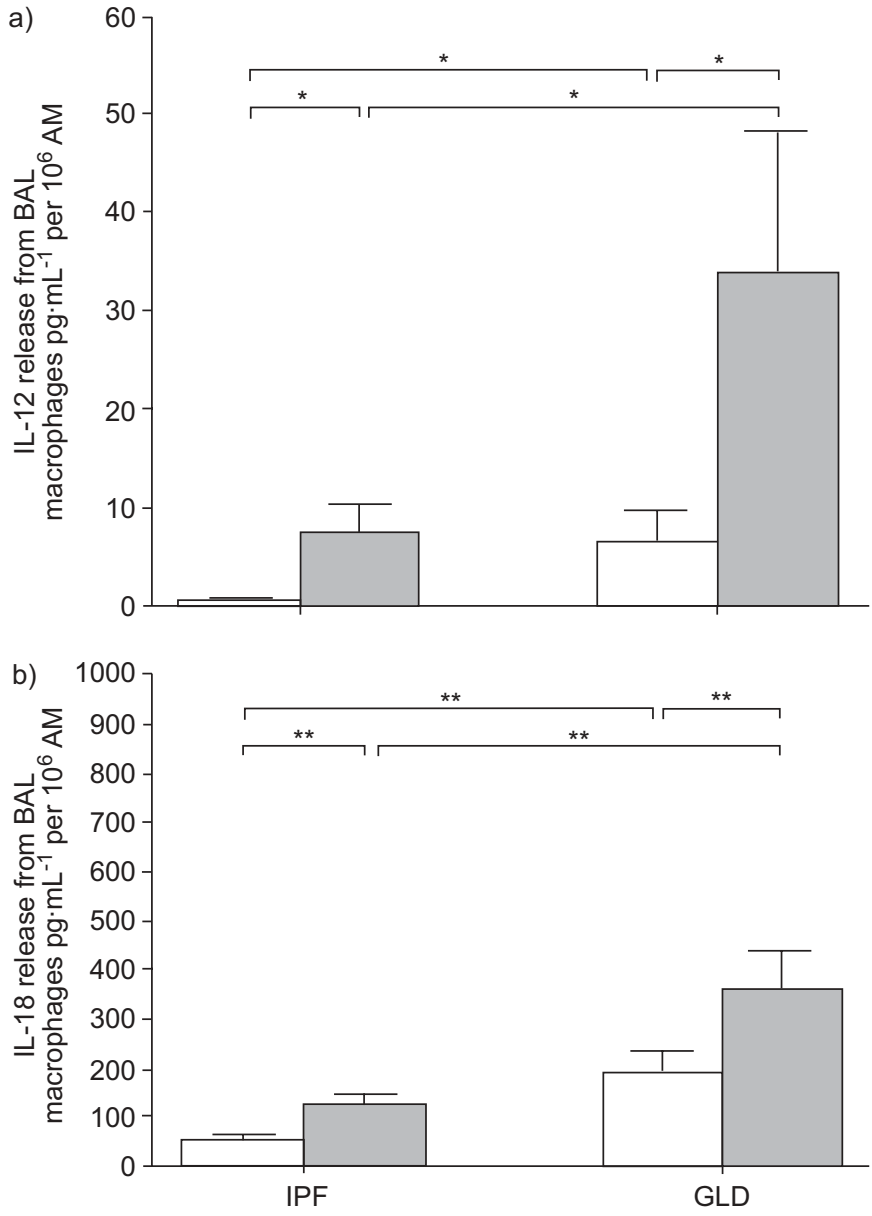

FIGURE 2. Spontaneous $(\square)$ and lipopolysaccharide-stimulated $(\square)$ production of a) interleukin (IL)-12 and b) IL-18 from bronchoalveolar lavage (BAL) alveolar macrophages (AM) in patients with idiopathic pulmonary fibrosis (IPF; $n=12$ ) and granulomatous lung disorders (GLD; sarcoidosis and hypersensitivity pneumonitis; $n=12) . *: p<0.05 ; * *: p<0.01$

(GLD) to allow statistically meaningful comparisons. After $24 \mathrm{~h}$ of culture, the spontaneous production of IL-12 (mean \pm SEM) from BAL macrophages was significantly lower in IPF than in GLD $\left(0.7 \pm 0.07\right.$ versus $7.5 \pm 2.9 \mathrm{pg} \cdot \mathrm{mL}^{-1}$ per $10^{6}$ AM, $\mathrm{p}<0.05)$. Similarly, the level of IL-18 was significantly lower in IPF than in GLD $(55.2 \pm 10.7$ versus $188.7 \pm 44.7 \mathrm{pg} \cdot \mathrm{mL}^{-1}$ per $10^{6} \mathrm{AM}, \mathrm{p}<0.01$; fig. 2 ).

The LPS-stimulated production of IL-12 and IL-18 was significantly higher than the spontaneous release in both groups $(\mathrm{p}<0.05$ and $\mathrm{p}<0.01$, respectively). The LPS-stimulated production of IL-12 and IL-18 was also significantly lower in IPF than in GLD (IL-12: $6.5 \pm 3.2$ versus $33.8 \pm 13.7 \mathrm{pg} \cdot \mathrm{mL}^{-1}$ per $10^{6} \mathrm{AM}, \mathrm{p}<0.05$; IL-18: $123.1 \pm 24.2$ versus $358.8 \pm 81.7 \mathrm{pg} \cdot \mathrm{mL}^{-1}$ per $10^{6}$ AM, $\mathrm{p}<0.01$; fig. 2).

\section{Correlations between HO-1 expression and BAL cell differentials}

In IPF patients, there were only weak correlations between the score of HO-1 positive AMs and the percentage of neutrophils $(\mathrm{r}=0.40, \mathrm{p}=0.052)$ or eosinophils $(\mathrm{r}=0.33, \mathrm{p}=0.111)$ in the BALF, statistically of borderline or no significance. In

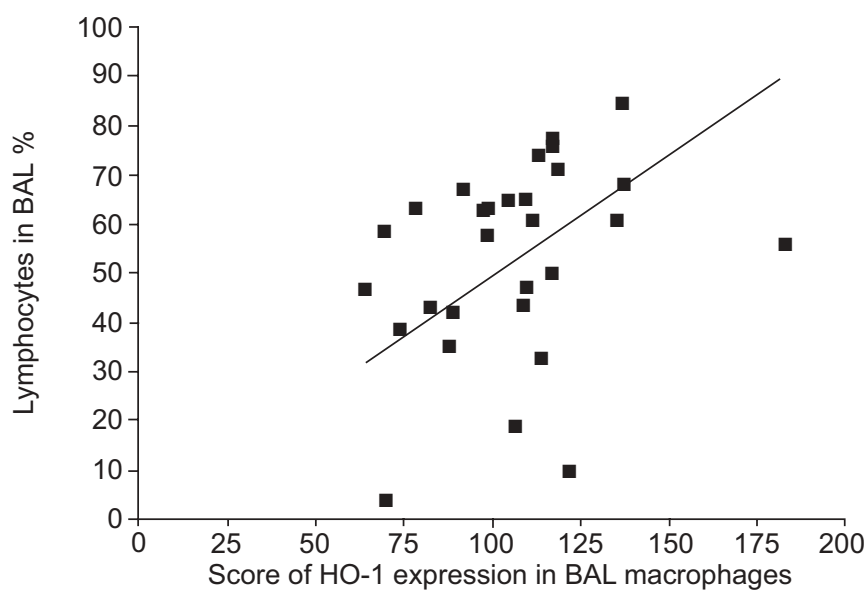

FIGURE 3. Correlation of scores of haem oxygenase (HO)-1 expression in macrophages with percentage of lymphocytes from bronchoalveolar lavage (BAL) in granulomatous lung disorders (sarcoidosis $n=16$, hypersensitivity pneumonitis $n=14) . r=0.38, p<0.05$.

sarcoidosis and HP patients, the score of HO-1 positive AMs showed a significant correlation with the percentage of lymphocytes $(r=0.38, p<0.05)$ in the BALF (fig. 3$)$.

\section{Correlations between HO-1 expression and cytokine release from $B A L$ macrophages}

In IPF patients, positive correlations were found between the score of $\mathrm{HO}-1$ positive AMs and the spontaneous production of IL-12 and IL-18 in supernatants of AM cultures ( $r=0.58$, $p<0.05$ and $r=0.60, p<0.05$, respectively; fig. 4). No further correlations were found between $\mathrm{HO}-1$ expression and cytokine production either in IPF or in GLD patients.

\section{Correlations between HO-1 expression and lung function}

The score of HO-1 failed to show any correlation with the lung function values of the patients with interstitial lung disease.

\section{Total bilirubin and ferritin in the macrophage culture supernatants}

Total bilirubin was significantly lower in the AM culture supernatants of IPF patients than in sarcoidosis and HP patients $\left(3.6 \pm 0.4\right.$ versus $9.9 \pm 2.1 \mathrm{mg} \cdot \mathrm{mL}^{-1}$ per $10^{6} \mathrm{AM}$, $\mathrm{p}<0.05$ ), whereas the ferritin concentration was not different between IPF and granulomatous lung disorders $(170 \pm 34$ versus $189 \pm 35 \mathrm{ng} \cdot \mathrm{mL}^{-1}$ per $10^{6} \mathrm{AM}, \mathrm{p}>0.05$; fig. 5).

\section{DISCUSSION}

The present study showed that the expression of $\mathrm{HO}-1$ by AMs is reduced in patients with IPF. In these patients, the score of HO-1 expression in macrophages correlated positively with IL12 and IL-18 release from AM in vitro. There was no difference in the AM HO-1 expression between patients with GLD (sarcoidosis and HP) and the control subjects. The present results are in agreement with an immunohistochemical study which showed that HO-1 expression was higher in newly diagnosed sarcoidosis than in UIP patients [27]. The present study's limitation was due to the causality of the decreased HO-1 expression in IPF patient AMs not being determined. 

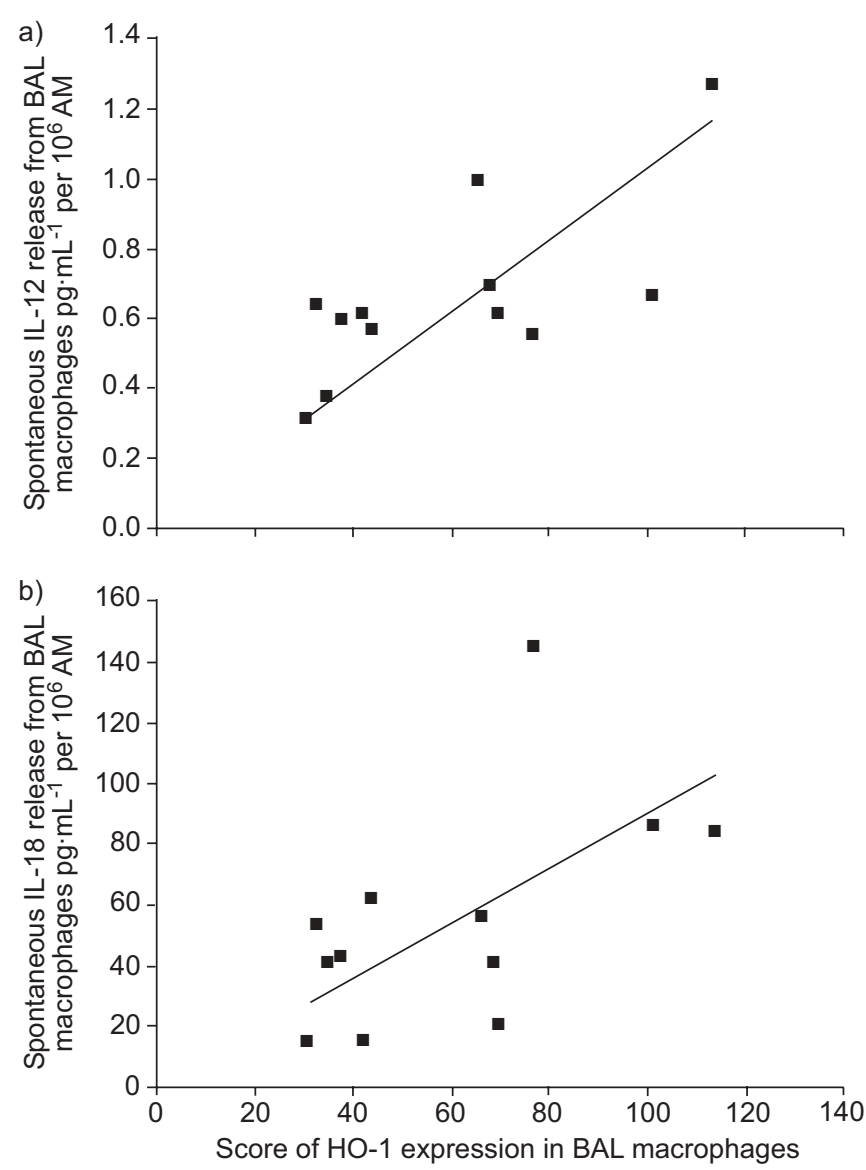

FIGURE 4. Correlations of spontaneous release of a) interleukin (IL)-12 and b) IL-18 with score of haem oxygenase (HO)-1 expression in alveolar macrophages (AM) from bronchoalveolar lavage (BAL) in patients with idiopathic pulmonary fibrosis. a) $r=0.58, p<0.05$; b) $r=0.60, p<0.05$.

HO-1, as an important mediator of cytoprotection against oxidative injury, may be implicated in the pathogenesis of IPF. Basal expression of $\mathrm{HO}-1$ has been described in hamster fibroblasts and was associated with resistance to hyperoxia [28]. In a murine model of pulmonary fibrosis, HO-1 was found to be one of the most differentially expressed genes as revealed by cDNA array analysis, and increased $\mathrm{HO}-1$ protein expression was also observed [29]. A potential salutary effect of HO-1 in the pathogenesis of pulmonary fibrosis has been proposed. TSUBURAI et al. [30] reported that adenovirusmediated transfer of the HO-1 gene can attenuate the intensity of bleomycin-induced pulmonary fibrosis in a murine model. Conversely, zinc-deuteroporphyrin (Zndtp), a chemical inhibitor of $\mathrm{HO}$ activity, has recently been shown to be protective in a bleomycin-induced lung fibrosis model by decreasing total lung collagen and the degree of extracellular matrix deposition and increasing glutathione levels [31]. How can the different effects of the two studies be explained, one showing a protection against bleomycin-induced fibrosis by $\mathrm{HO}-1$ overexpression [30], the other a similar protection by $\mathrm{HO}$ inhibition [31]? First, the antioxidative properties that are intrinsic to the metalloporphyrin Zndtp itself may act to restore the alveolar antioxidant capacity. Secondly, the role of $\mathrm{HO}-1$ in tissue injury
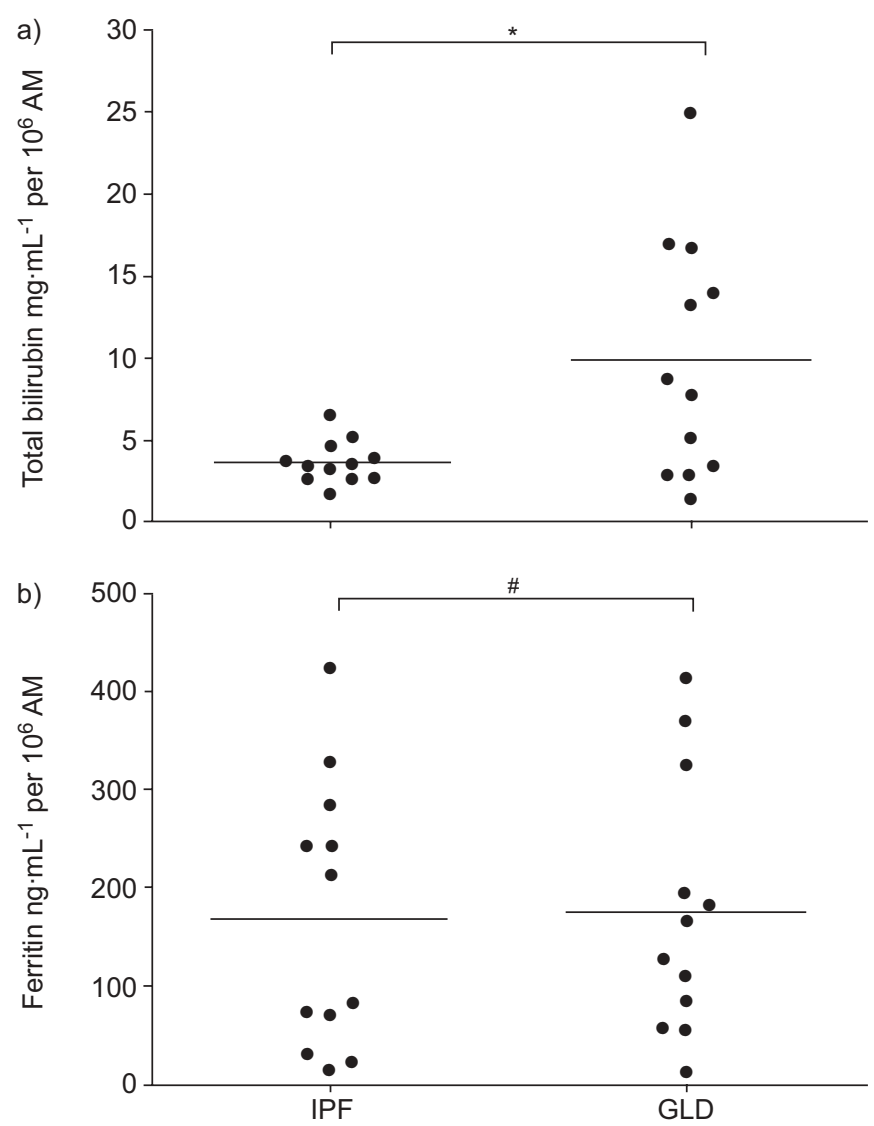

FIGURE 5. The concentration of a) total bilirubin and b) ferritin in the alveolar macrophage (AM) culture supernatants of patients with idiopathic pulmonary fibrosis (IPF; $n=12$ ) and granulomatous lung disorders (GLD; sarcoidosis and hypersensitivity pneumonitis; $n=12$ ). *: $p<0.05$; ${ }^{*}: p>0.05$.

is complex and is determined by its local concentration and temporal prominence $[16,32]$. In the study by TsubURAI et al. [30] HO-1 protein expression was induced before bleomycin treatment, whereas, in the study by ATZORI et al. [31], Zndtp was given after the initial inflammatory phase. This may account for the reduced fibrosis in both the overexpression study and the inhibitory study, suggesting that HO may play different roles in the early inflammatory phase and in the later fibroproliferative phase of bleomycin-induced lung toxicity. However, it is important to note that the bleomycin model does not completely correlate with human IPF. The bleomycin model results from acute lung injury leading to early fibrosis, whereas clinically diagnosed IPF patients are mostly in the late fibrotic stage, with little evidence of inflammation.

The role of the observed downregulation of HO-1 in the pathogenesis of IPF may be explained in two ways: it may either serve as an adaptive mechanism to protect the tissue against harmful effects of haem degradation products, or it may indicate that loss of protection against oxidative stress is involved in the pathogenetic mechanisms of IPF. There is more evidence to support the second assumption. Similarly to HO-1, the expression of MnSOD, an important superoxide radicalscavenging antioxidative enzyme, has been shown to be increased in inflammatory disorders and GLD but to be low in the fibrotic lesions of IPF lungs [12]. Likewise, other 
antioxidants and detoxification enzymes including catalase, glutamate cysteine ligase, glutaredoxin and thioredoxin show similar changes in opposite directions, i.e. upregulation in acute inflammation models or diseases and downregulation in chronic fibrosis models or diseases [2, 10-12].

Although the role of inflammation in the pathogenesis of IPF remains controversial, evidence supports an imbalance of the Th1 (interferon (IFN)- $\gamma$, IL-12 and IL-18) and Th2 (IL-4 and IL-5) cytokine profiles in favour of the Th2 profile, which may contribute to the accumulation of collagen [22]. Sarcoidosis and HP are characterised by a Th1-type immune response which leads to exaggerated inflammation, setting the stage for granuloma formation and only rarely for the development of irreversible remodelling of the lung tissue and fibrosis [23, 24]. IL-12 and IL-18 are key cytokines involved in regulating the balance between Th1 and Th2 profiles. The results of the present study showed that both the spontaneous and LPS-stimulated production of IL-12 and IL-18 from BAL macrophages was significantly higher in sarcoidosis and HP patients than in IPF patients. This is consistent with the assumption that sarcoidosis and HP are Th1 diseases and IPF is a Th2 disease [22-24].

Recently, IL-12 has been shown to attenuate bleomycininduced murine lung fibrosis by an IFN- $\gamma$ dependent manner [33]. In the present study, the score of $\mathrm{HO}-1$ expression in AMs failed to correlate with the production of IL-12 and IL-18 in patients with sarcoidosis and HP. However, in IPF patients, low HO-1 expression in AMs correlated with low spontaneous production of IL-12 and IL-18, indicating that the switch to the Th2 pattern was most profound in those IPF patients with the lowest HO-1 expression. The involvement of $\mathrm{HO}-1$ in the regulation of inflammation has been shown in a number of diseases and animal models. Both $\mathrm{HO}-1$ and $\mathrm{CO}$ can decrease the LPS-induced expression of pro-inflammatory cytokines, including tumour necrosis factor- $\alpha$, IL- $1 \beta$ and macrophage inflammatory protein- $1 \beta$, and augment the expression of the anti-inflammatory cytokine IL-10 [34, 35]. IL-10 mediated protection against LPS-induced septic shock in mice can be significantly attenuated by co-treatment with the $\mathrm{HO}$ inhibitor zinc-protoporphyrin [35]. Exogenous administration of $\mathrm{CO}$ to mice or macrophages leads to a decrease in the production of IL-6 [36]. To summarise, HO-1 is involved in the inflammatory modulation of lung diseases, the major effect being downregulation of pro-inflammatory cytokines. Conversely, transforming growth factor- $\beta 1$, an important pro-fibrotic cytokine, is able to downregulate HO-1 in the rat lung [37]. Diminished expression of HO-1 is probably associated with a cellular micro-environment that promotes fibrosis.

Although the mechanisms underlying the cytoprotective function of HO-1 remain unclear, the haem degradation products including bilirubin, $\mathrm{CO}$ and iron-induced ferritin seem to be mediators of the effects of HO-1 activation [13]. In asthmatic patients, exhaled $\mathrm{CO}$ and sputum bilirubin levels have been shown to be elevated in agreement with increased HO-1 staining [15, 38]. Increased ferritin staining in AMs was observed in cystic fibrosis lungs compared with normal lungs, and the number of ferritin-stained macrophages correlated with the amount of HO-1 staining [17]. Inhaled CO had suppressive effects on fibrosis in bleomycin models of lung injury [39]. Bilirubin is also regarded as a powerful antioxidant substance in vitro [40] and a very effective physiological antioxidant in vivo [41]. Bilirubin is not only an important antioxidant in serum, but may also play an important role in pulmonary fibrosis. This was suggested by a recent study where hyperbilirubinaemia was shown to attenuate bleomycin-induced pulmonary fibrosis [42]. In the present study, total bilirubin was significantly lower in the AM culture supernatants of IPF patients than in those of sarcoidosis and HP patients, similar to the changes observed for the HO-1 expression in AM.

In conclusion, the present study showed that haem oxygenase-1, an important antioxidant, is depleted in bronchoalveolar macrophages of idiopathic pulmonary fibrosis patients. Given the fact that murine models have been reported suggesting that overexpression of haem oxygenase- 1 attenuates bleomycininduced lung injury [29], the current study translates the findings of murine models into the human realm. This has not been previously reported but is necessary to advance research in this field. The findings support the hypothesis of an oxidantantioxidant imbalance in the pathogenesis of idiopathic pulmonary fibrosis. Targeting haem oxygenase- 1 expression in the lungs of patients with idiopathic pulmonary fibrosis may be a new therapeutic approach, but further research is warranted to elucidate the complex beneficial or harmful role of haem oxygenase- 1 in interstitial lung disease.

\section{REFERENCES}

1 American Thoracic Society, European Respiratory Society. Idiopathic pulmonary fibrosis: diagnosis and treatment, international consensus statement. Am J Respir Crit Care Med 2000; 161: 646-664.

2 Kinnula VL, Fattman CL, Tan RJ, Oury TD. Oxidative stress in pulmonary fibrosis: a possible role for redox modulatory therapy. Am J Respir Crit Care Med 2005; 172: 417-422.

3 Cantin AM, North SL, Fells GA, Hubbard RC, Crystal RG. Oxidant-mediated epithelial cell injury in idiopathic pulmonary fibrosis. J Clin Invest 1987; 79: 1665-1673.

4 Nathan CF, Brukner LH, Silverstein SC, Cohn ZA. Extracellular cytolysis by activated macrophages and granulocytes. I. Pharmacologic triggering of effector cells and the release of hydrogen peroxide. J Exp Med 1979; 149: 84-99.

5 Kuwano K, Nakashima N, Inoshima I, et al. Oxidative stress in lung epithelial cells from patients with idiopathic interstitial pneumonias. Eur Respir J 2003; 21: 232-240.

6 Lenz AG, Costabel U, Maier KL. Oxidized BAL fluid proteins in patients with interstitial lung diseases. Eur Respir J 1996; 9: 307-312.

7 Maier K, Leuschel L, Costabel U. Increased levels of oxidized methionine residues in bronchoalveolar lavage fluid proteins from patients with idiopathic pulmonary fibrosis. Am Rev Respir Dis 1991; 143: 271-274.

8 Montuschi P, Ciabattoni G, Paredi P, et al. 8-Isoprostane as a biomarker of oxidative stress in interstitial lung diseases. Am J Respir Crit Care Med 1998; 158: 1524-1527.

9 Meyer A, Buhl R, Magnussen H. The effect of oral Nacetylcysteine on lung glutathione levels in idiopathic pulmonary fibrosis. Eur Respir J 1994; 7: 431-436.

10 Tiitto L, Kaarteenaho-Wiik R, Sormunen R, et al. Expression of the thioredoxin system in interstitial lung disease. J Pathol 2003; 201: 363-370. 
11 Peltoniemi M, Kaarteenaho-Wiik R, Säily $\mathrm{M}$, et al. Expression of glutaredoxin is highly cell specific in human lung and is decreased by transforming growth factor- $\beta$ in vitro and in interstitial lung diseases in vivo. Hum Pathol 2004; 35: 1000-1007.

12 Lakari E, Pääkkö P, Pietarinen-Runtti P, Kinnula VL. Manganese superoxide dismutase and catalase are coordinately expressed in the alveolar region in chronic interstitial pneumonias and granulomatous diseases of the lung. Am J Respir Crit Care Med 2000; 161: 615-621.

13 Morse D, Choi AM. Heme oxygenase-1: from bench to bedside. Am J Respir Crit Care Med 2005; 172: 660-670.

14 Maestrelli P, El Messlemani AH, De Fina O, et al. Increased expression of heme oxygenase (HO)-1 in alveolar spaces and HO-2 in alveolar walls of smokers. Am J Respir Crit Care Med 2001; 164: 1508-1513.

15 Horváth I, Donnelly LE, Kiss A, Paredi P, Kharitonov SA, Barnes PJ. Raised levels of exhaled carbon monoxide are associated with an increased expression of heme oxygenase- 1 in airway macrophages in asthma: a new marker of oxidative stress. Thorax 1998; 53: 668-672.

16 Lee PJ, Alam J, Sylvester SL, Inamdar N, Otterbein L, Choi AM. Regulation of heme oxygenase-1 expression in vivo and in vitro in hyperoxic lung injury. Am J Respir Cell Mol Biol 1996; 14: 556-568.

17 Zhou H, Lu F, Latham C, Zander DS, Visner GA. Heme oxygenase-1 expression in human lungs with cystic fibrosis and cytoprotective effects against Pseudomonas aeruginosa in vitro. Am J Respir Crit Care Med 2004; 170: 633-640.

18 Lu F, Zander DS, Visner GA. Increased expression of heme oxygenase-1 in human lung transplantation. J Heart Lung Transplant 2002; 21: 1120-1126.

19 Maestrelli P, Páska C, Saetta M, et al. Decreased haem oxygenase- 1 and increased inducible nitric oxide synthase in the lung of severe COPD patients. Eur Respir J 2003; 21: 971-976.

20 Moller DR, Forman JD, Liu MC, et al. Enhanced expression of IL-12 associated with Th1 cytokine profiles in active pulmonary sarcoidosis. J Immunol 1996; 156: 4952-4960.

21 Chen B, Tong Z, Nakamura S, Costabel U, Guzman J. Production of IL-12, IL-18 and TNF- $\alpha$ by alveolar macrophages in hypersensitivity pneumonitis. Sarcoidosis Vasc Diffuse Lung Dis 2004; 21: 199-203.

22 Majumdar S, Li D, Ansari T, et al. Different cytokine profiles in cryptogenic fibrosing alveolitis and fibrosing alveolitis associated with systemic sclerosis: a quantitative study of open lung biopsies. Eur Respir J 1999; 14: 251-257.

23 Hunninghake GW, Costabel U, Ando M, et al. ATS/ERS/ WASOG statement on sarcoidosis. American Thoracic Society/European Respiratory Society/World Association of Sarcoidosis and other Granulomatous Disorders. Sarcoidosis Vasc Diffuse Lung Dis 1999; 16: 149-173.

24 Yamasaki H, Ando M, Brazer W, Center DM, Cruikshank WW. Polarized type 1 cytokine profile in bronchoalveolar lavage $\mathrm{T}$ cells of patients with hypersensitivity pneumonitis. J Immunol 1999; 163: 3516-3523.

25 Klech H, Pohl W. Technical recommendations and guidelines for bronchoalveolar lavage (BAL). Report of the European Society of Pneumology Task Group. Eur Respir J 1989; 2: 561-585.
26 Costabel U, Bross KJ, Matthys H. The immunoperoxidase slide assay. A new method for the demonstration of surface antigens on bronchoalveolar lavage cells. Bull Eur Physiopathol Respir 1985; 21: 381-387.

27 Lakari E, Pylkäs P, Pietarinen-Runtti P, Pääkkö P, Soini Y, Kinnula VL. Expression and regulation of hemeoxygenase 1 in healthy human lung and interstitial lung disorders. Hum Pathol 2001; 32: 1257-1263.

28 Dennery PA, Wong HE, Sridhar KJ, Rodgers PA, Sim JE, Spitz DR. Differences in basal and hyperoxia-associated HO expression in oxidant-resistant hamster fibroblasts. Am J Physiol 1996; 271: L672-L679.

29 Kaminski N, Allard JD, Pittet JF, et al. Global analysis of gene expression in pulmonary fibrosis reveals distinct programs regulating lung inflammation and fibrosis. Proc Natl Acad Sci USA 2000; 97: 1778-1783.

30 Tsuburai T, Suzuki M, Nagashima Y, et al. Adenovirusmediated transfer and overexpression of heme oxygenase 1 cDNA in lung prevents bleomycin-induced pulmonary fibrosis via a Fas-Fas ligand-independent pathway. Hum Gene Ther 2002; 13: 1945-1960.

31 Atzori L, Chua F, Dunsmore SE, et al. Attenuation of bleomycin induced pulmonary fibrosis in mice using the heme oxygenase inhibitor Zn-deuteroporphyrin IX-2,4bisethylene glycol. Thorax 2004; 59: 217-223.

32 Suttner DM, Dennery PA. Reversal of HO-1 related cytoprotection with increased expression is due to reactive iron. FASEB J 1999; 13: 1800-1809.

33 Keane MP, Belperio JA, Burdick MD, Strieter RM. IL-12 attenuates bleomycin-induced pulmonary fibrosis. Am J Physiol Lung Cell Mol Physiol 2001; 281: L92-L97.

34 Otterbein LE, Bach FH, Alam J, et al. Carbon monoxide has anti-inflammatory effects involving the mitogen-activated protein kinase pathway. Nat Med 2000; 6: 422-428.

35 Lee TS, Chau LY. Heme oxygenase-1 mediates the antiinflammatory effect of interleukin-10 in mice. Nat Med 2002; 8: 240-246.

36 Dyck D, Otterbein LE, Choi AMK. Carbon monoxide attenuates lipopolysaccharide-induced cytokine expression of IL-6 and IL-1 $\beta$. Am J Respir Crit Care Med 2000; 161: A664.

37 Pellacani A, Wiesel P, Sharma A, et al. Induction of heme oxygenase- 1 during endotoxemia is downregulated by transforming growth factor- $\beta 1$. Circ Res 1998; 83: 396-403.

38 Zayasu K, Sekizawa K, Okinaga S, Yamaya M, Ohrui T, Sasaki H. Increased carbon monoxide in exhaled air of asthmatic patients. Am J Respir Crit Care Med 1997; 156: 1140-1143.

39 Zhou Z, Song R, Fattman CL, et al. Carbon monoxide suppresses bleomycin-induced lung fibrosis. Am J Pathol 2005; 166: 27-37.

40 Stocker R, Yamamoto Y, McDonagh AF, Glazer AN, Ames BN. Bilirubin is an antioxidant of possible physiological importance. Science 1987; 235: 1043-1046.

41 Dennery PA, McDonagh AF, Spitz DR, Rodgers PA, Stevenson DK. Hyperbilirubinemia results in reduced oxidative injury in neonatal Gunn rats exposed to hyperoxia. Free Radic Biol Med 1995; 19: 395-404.

42 Wang HD, Yamaya M, Okinaga S, et al. Bilirubin ameliorates bleomycin-induced pulmonary fibrosis in rats. Am J Respir Crit Care Med 2002; 165: 406-411. 